\title{
Ictal magnetoencephalographic study in a patient with ring 20 syndrome
}

\author{
N Tanaka, K Kamada, F Takeuchi
}

J Neurol Neurosurg Psychiatry 2004;75:488-490. doi: 10.1136/jnnp.2003.020438

\begin{abstract}
Objective: To report the ictal magnetoencephalography (MEG) in a patient with ring chromosome 20 mosaicism, a rare chromosomal anomaly associated with intractable epilepsy.

Methods: MEG and simultaneous EEG were recorded with a 204 channel whole head MEG system. Ten habitual seizures occurred during the acquisition, which was done twice. The equivalent current dipoles (ECDs) for ictal discharges on MEG were calculated using a single dipole model. The ECDs were superimposed on a magnetic resonance image.

Results: During the seizures, EEG showed prolonged bursts of 5-6 Hz high voltage slow waves with spike components, dominantly in the bilateral frontal region. MEG showed epileptiform discharges corresponding to the ictal EEG. Ictal discharges on MEG were dominant in the frontal area in the initial portion, and then spread in the bilateral temporal area in the middle of the seizure. ECDs obtained from the spikes of the initial portion were clustered in the medial frontal lobe. Conclusions: The source of the ictal MEG was localised in the medial frontal lobe. The findings suggest that the mechanism underlying epilepsy in this case might be similar to medial frontal lobe epilepsy. Ictal MEG is a valuable tool for detecting the site of seizure onset.
\end{abstract}

$M$ any types of chromosomal anomaly are known to be associated with epilepsy. Patients with ring chromosome 20 have the so called ring 20 syndrome, which is characterised by mental retardation, behavioural problems, and intractable epilepsy. ${ }^{1}$ Several researchers have reported that ictal electroencephalography (EEG) in patients with ring 20 syndrome shows a particular pattern of prolonged high voltage slow waves or spike and slow wave complexes, dominantly in the bilateral frontal region..$^{2-4}$ However, the origin of the ictal discharge has not been determined.

Magnetoencephalography (MEG) is a powerful tool for investigating epileptogenic foci and has been used for localising the source of interictal epileptic discharges. The value of ictal MEG recording has recently been reported. ${ }^{5-8}$

In the present study, we simultaneously recorded ictal MEG and EEG in a patient with ring 20 syndrome. Dipole source localisation was calculated on magnetic fields for ictal discharges.

\section{CASE REPORT}

The 18 year old male patient was born after an uncomplicated pregnancy. Psychomotor development during early childhood was apparently normal. Seizure onset was at eight years. He had experienced several seizures a week, during which he had clouding of consciousness lasting around 30 seconds. Generalised tonic-clonic seizures also occurred once or twice a year. He was treated with carbamazepine, valproate, phenytoin, and phenobarbitone (phenobarbital), but seizures were only partially controlled. He entered high school at the age of 15 years, but learning disability was evident at that time. He therefore left the school two years later. At the age of 18 years, he was admitted to the department of psychiatry and neurology of our hospital.

The interictal EEG showed frequent anterior dominant 5-6 $\mathrm{Hz}$ waves. The ictal EEG showed bursts of 5-6 Hz high voltage slow waves with spike components, dominantly in the bilateral frontal region. There were no abnormal findings on magnetic resonance imaging (MRI). Single photon emission computed tomography with ${ }^{123}$ I-IMP showed hypoperfusion in the right frontal lobe. On the WAIS-R, he scored an IQ of 56 (verbal IQ 60; performance IQ 66). The cytogenetic studies showed the presence of ring chromosome in $6.7 \%$ of the lymphocytes studied: $46, \mathrm{XY}, \mathrm{r}(20)(\mathrm{pl}$ q 13.3$)$ [2]/46,XY[28].

\section{MEG FINDINGS}

We recorded MEG and simultaneous EEG twice. The recording was done for 30 minutes in each acquisition. MEG data were recorded with a 204 channel whole head MEG system (4D-Neuroimage, Helsinki, Finland) in a magnetically shielded chamber. An EEG was obtained simultaneously, employing scalp electrodes according to the international 10-20 system. The sampling rate of MEG and EEG was $600 \mathrm{~Hz}$. In data analysis, MEG data were filtered digitally at a bandpass width of 0.5 to $30 \mathrm{~Hz}$. For magnetic source localisation, equivalent current dipoles (ECD) were calculated according to a single dipole model. ECDs with a goodness of fit better than $80 \%$ were considered adequate.

During the examinations, 10 typical seizures occurred, and the ictal MEG data were successfully recorded. The patient was unresponsive and could not obey simple commands during these seizures. He also could not recall the events occurring during the seizures. Raw MEG data and the simultaneous EEG during a typical seizure are shown in fig lA. The EEG showed bursts of 5-6 Hz high voltage slow waves with spike components lasting over 30 seconds, predominantly in the bilateral frontal area. The MEG showed a waveform that was similar in appearance to the EEG burst. In the initial portion of the ictal MEG, the discharges were dominant in the frontal region. In the middle part, the MEG spikes were dominant in the bilateral temporal area. In all, 36 spikes from the initial parts of 10 seizures were used for dipole analysis. We obtained 21 ECDs with a goodness of fit better than $80 \%$ from these spikes. ECDs with the highest goodness of fit value from each seizure were selected and superimposed on the MRI of the patient. These ECDs formed

\footnotetext{
Abbreviations: ECD, equivalent current dipole; MEG, magnetoencephalography; WAIS-R, Wechsler adult intelligence scale, revised
} 


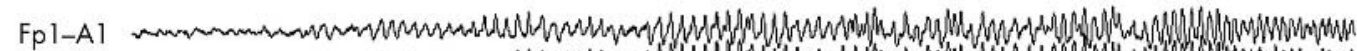

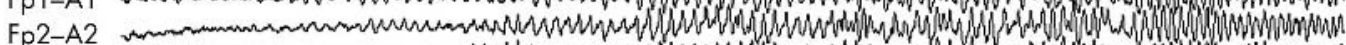

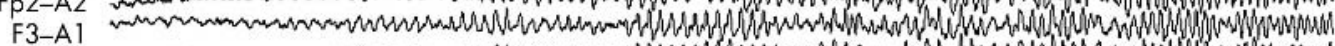

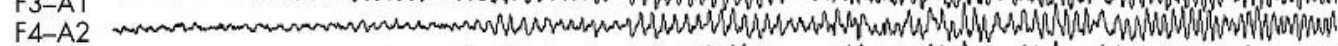

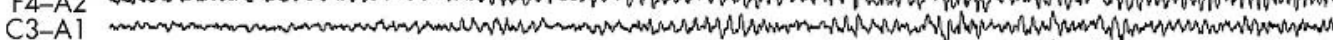

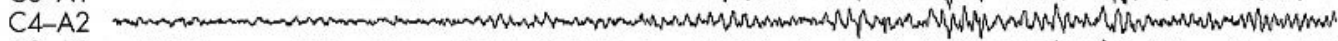

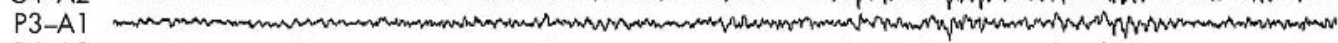

P4-A2 rim

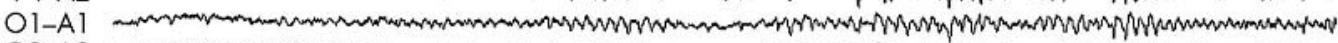

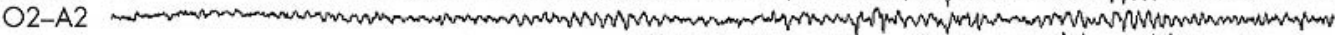

EEG F7-A1 - Them

F8-A2

T3-A1 r.mon

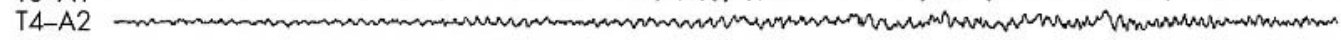

T5-A1 200

T6-A2 con

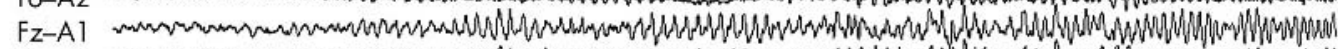

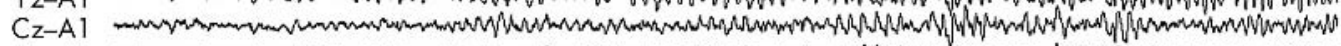

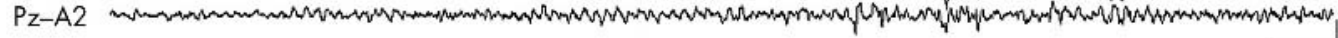

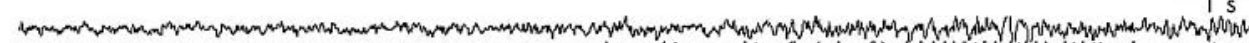

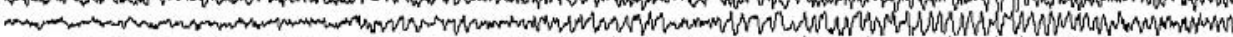

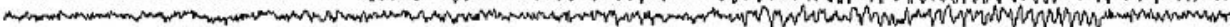

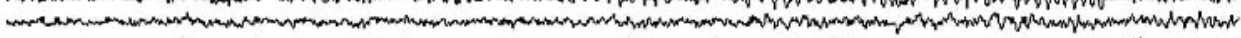
(n)

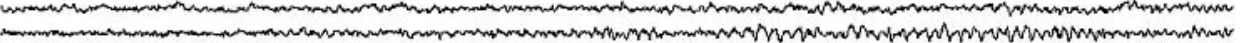

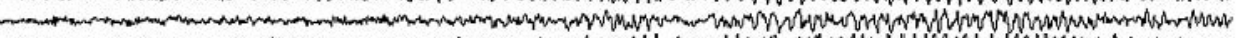

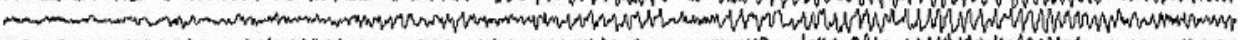

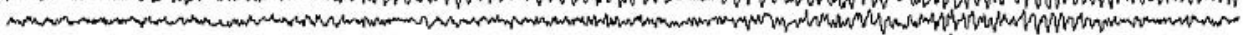

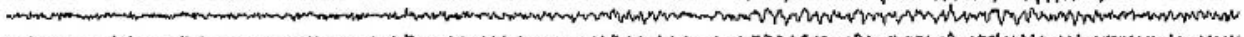
Nom Non -

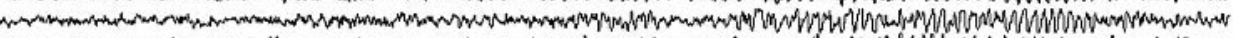

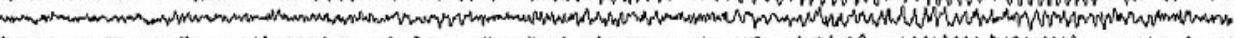

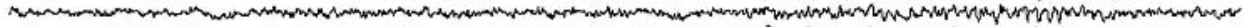
whom

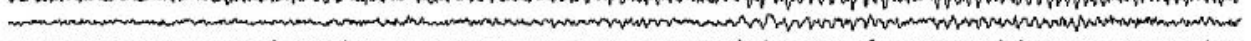

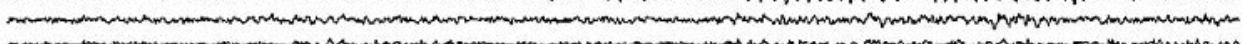

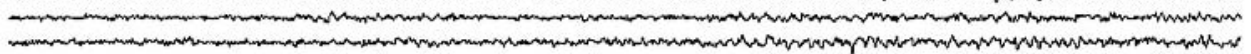

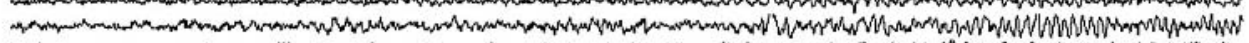

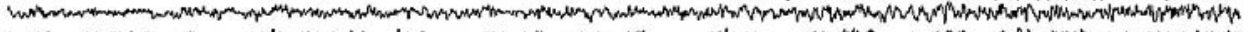
mom

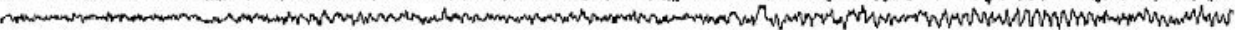

B
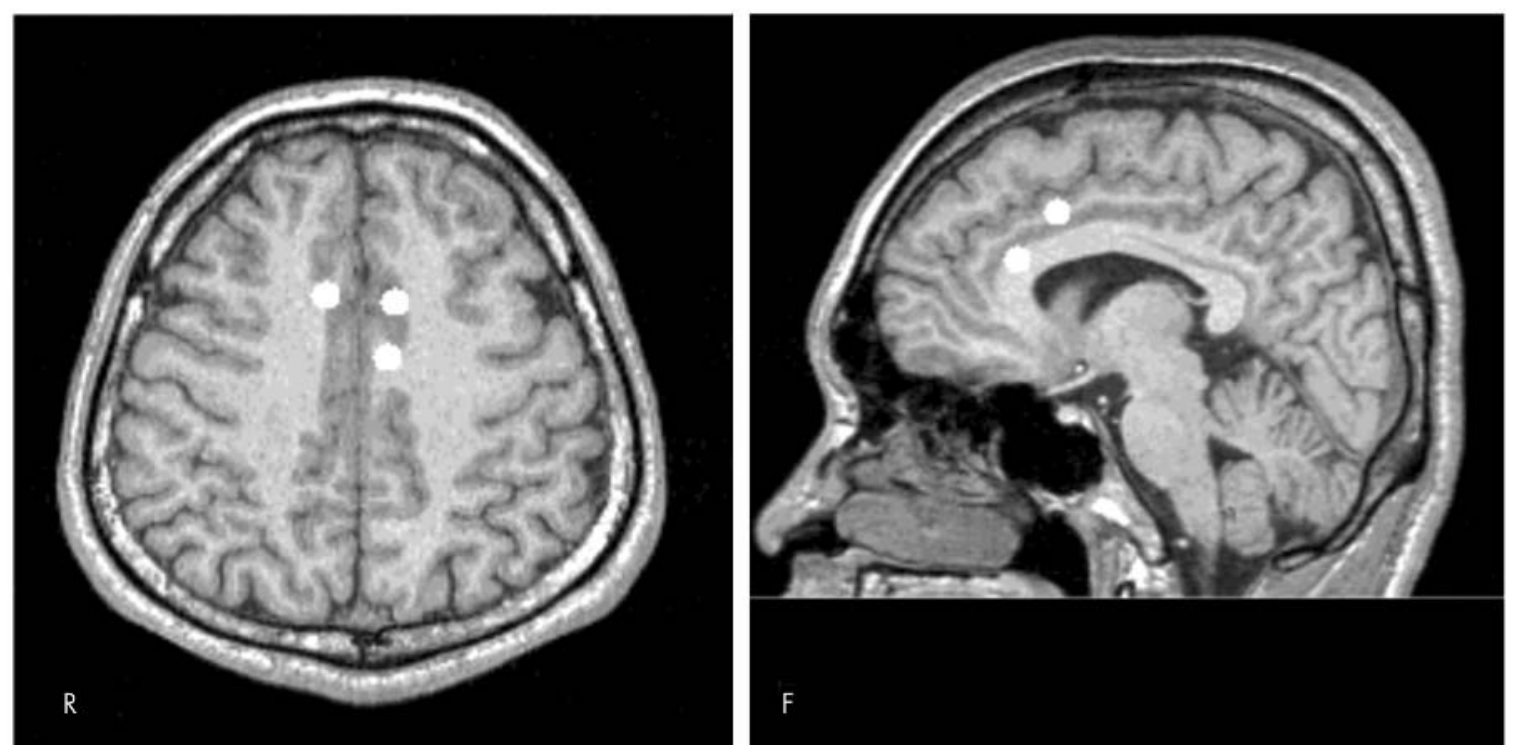

Figure 1 (A) Ictal magnetoencephalography (MEG) recorded from 36 channels covering the bilateral frontal area and simultaneously recorded electroencephalogram. MEG showed ictal discharges corresponding to the ictal EEG. (B) Equivalent current dipoles (ECDs) calculated from the spikes in the initial portion of ictal MEG. White circles represent the positions of ECDs superimposed on the magnetic resonance image of the patient. ECDs were clustered in the medial frontal lobe. 
a cluster in the medial frontal lobe, and the dipole source localisation in different seizures also agreed, confirmed this positioning (fig 1B). The ECDs calculated from the spikes in the middle of the ictal MEG had a goodness of fit of less than $80 \%$.

\section{DISCUSSION}

In this case, there was ring chromosome 20 mosaicism and the electroclinical features were typical of ring 20 syndrome. The clinical pattern of the habitual seizures was characterised by clouded consciousness lasting over 30 seconds without any motor symptoms or convulsion. Previous studies of ring 20 syndrome have shown that there are different types of clinical seizure, most of which are accompanied by loss of consciousness with some lateralised signs, and are considered to be complex partial seizures. ${ }^{23}$ On the other hand, Holopainen et al reported a case of ring 20 syndrome who showed clinical features resembling absence seizures. ${ }^{9}$ The seizure pattern in our case appears similar.

The ictal MEG showed that the frontal dominant ictal discharges corresponding to the ictal EEG at the beginning of the seizures. The clustered ECDs in the medial frontal lobecalculated from the initial part of the ictal MEG-showed that the site of ictal onset was localised in this area. In previous reports of EEG in ring 20 syndrome, the origin of the ictal discharges was expected to be in the frontal lobe. ${ }^{2-4}$ However, source analysis of these discharges was quite difficult until whole head MEG became available, and the part of the frontal lobe associated with the onset of the seizures has not been determined. Our dipole source localisation results confirmed that the generators of the ictal discharges were localised in the medial region. Shiraishi et al reported that the sources of MEG spikes corresponding to bilateral frontal discharges on EEG in patients with frontal lobe epilepsy were localised to the medial frontal cortex. ${ }^{10}$ Our EEG and MEG results agree with that analysis.

Bancaud et al described how patients with frontal lobe epilepsy whose ictal discharges initially affect the medial surface of the intermediate frontal region produce a type of complex partial seizure similar to absence seizures in their electroclinical features. ${ }^{11}$ This type of seizure may last for more than 10 seconds, and the ictal EEG shows bilateral, high amplitude, apparently symmetrical and synchronous spike-wave discharges. From the clinical seizure pattern and the clustering of the ECDs in the medial frontal cortex, we concluded that the type of seizure observed in our case was a complex partial seizure. Moreover, it appeared that the mechanism underlying the epilepsy in ring 20 syndrome might be similar to medial frontal lobe epilepsy.

We could not obtain ECDs with a goodness of fit value high enough to constitute an adequate source in the middle of the ictal MEG. After the beginning of the ictal MEG, epileptic discharges were widespread in the brain, and source localisation became difficult. In estimations of the origin of seizures by ictal MEG, spikes in the initial part should be used for analysis.

Recording and analysis of ictal MEG is difficult when head movements occur because of the seizure, as changes in head position make it impossible to calculate the correct dipole source localisation. In addition, artefacts caused by body movements during the seizure are obstacles to successful ictal MEG recording. ${ }^{78}$ Previous studies in larger patient groups showed that localisation of the ictal MEG was reliable as long as no movement occurred during the seizures. ${ }^{78}$ The seizures in our case were not accompanied by any motor symptoms, so we could avoid the problem of movement.

In conclusion, ictal MEG was highly valuable for investigating the site of seizure onset in this case of ring 20 syndrome.

\section{Authors' affiliations \\ N Tanaka, Department of Psychiatry and Neurology, Hokkaido \\ University School of Medicine, Sapporo, Japan \\ K Kamada, Department of Neurosurgery, Chitose City Hospital, Sapporo, Japan \\ F Takeuchi, Research Institute for Electronic Science, Hokkaido \\ University, Sapporo, Japan \\ Competing interests: none declared}

Correspondence to: Dr N Tanaka, Department of Psychiatry and Neurology, Hokkaido University School of Medicine, North 15, West 7, Sapporo 060-8638, Japan; naoro@med.hokudai.ac.jp

Received 9 June 2003

In revised form 6 August 2003

Accepted 6 August 2003

\section{REFERENCES}

1 Atkins L, Miller WL, Salam M. A ring-20 chromosome. J Med Genet 1972;9:377-80.

2 Inoue Y, Fujiwara T, Matsuda K, et al. Ring chromosome 20 and nonconvulsive status epilepticus. A new epileptic syndrome. Brain 1997; 120:939-53.

3 Kobayashi $K$, Inagaki $M$, Sasaki $M$, et al. Characteristic EEG findings in ring 20 syndrome as a diagnostic clue. Electroencephalogr Clin Neurophysiol 1998; 107:258-62.

4 Augastijn PB, Parra J, Wouters CH, et al. Ring chromosome 20 epilepsy syndrome in children: electroclinical features. Neurology 2001;57:1108-11.

5 Oishi M, Kameyama S, Morota N, et al. Fusiform gyrus epilepsy: the use of ictal magnetoencephalography. Case report. J Neurosurg 2002;97:200-4.

6 Oishi M, Otsubo H, Kameyama S, et al. Ictal magnetoencephalographic discharges from elementary visual hallucinations of status epileptics. I Neurol Neurosurg Psychiatry 2003:74:525-7.

7 Tilz C, Hummel C, Kettenmann B, et al. Ictal onset localization of epileptic seizures by magnetoencephalography. Acta Neurol Scand 2002;106:190-5.

8 Eliashiv DS, Elsas SM, Squires K, et al. Ictal magnetic source imaging as a localizing tool in partial epilepsy. Neurology 2002;59:1600-10.

9 Holopainen I, Penttinen M, Lakkala T, et al. Ring chromosome 20 mosaicism in a girl with complex partial seizures. Dev Med Child Neurol 1994;36:70-3.

10 Shiraishi H, Watanabe $Y$, Watanabe $M$, et al. Interictal and ictal magnetoencephalographic study in patients with medial frontal lobe epilepsy. Epilepsia 2001;42:875-82.

11 Bancaud J, Talairach J. Clinical semiology of frontal lobe seizures. In: Chauvel P, et al, eds. Advances in neurology, vol 57. New York: Raven Press, 1992:3-58. 